\title{
PAPER
}

\section{Localisation of the sensorimotor cortex during surgery for brain tumours: feasibility and waveform patterns of somatosensory evoked potentials}

\author{
J Romstöck, R Fahlbusch, O Ganslandt, C Nimsky, C Strauss
}

J Neurol Neurosurg Psychiatry 2002;72:221-229

See end of article for authors' affiliations

\section{Correspondence to:} Dr J Romstöck, Department of Neurosurgery, University of Erlangen-Nuremberg, Schwabachanlage 6, D-91054 Erlangen, Germany; romstoeck@ nch.imed.uni-erlangen.de

Received

5 January 2001

In revised form 3 June

2001

Accepted 20 April 2001

\begin{abstract}
Objective: Intraoperative localisation of the sensorimotor cortex using the phase reversal of somatosensory evoked potentials (SEPs) is an essential tool for surgery in and around the perirolandic gyri, but unsuccessful and perplexing results have been reported. This study examines the effect of tumour masses on the waveform characteristics and feasibility of SEP compared with functional neuronavigation and electrical motor cortex mapping.

Methods: In 230 patients with tumours of the sensorimotor region the SEP phase reversal of N20-P20 was recorded from the exposed cortex using a subdural grid or strip electrode. In one subgroup of 80 patients functional neuronavigation was performed with motor and sensory magnetic source imaging and in one subgroup of 40 patients the motor cortex hand area was localised by electrical stimulation mapping.

Results: The intraoperative SEP method was successful in $92 \%$ of all patients, it could be shown that the success rate rather depended on the location of the lesion than on preoperative neurological deficits. In 13\% of the patients with postcentral tumours no N20-P20 phase reversal was recorded but characteristic polyphasic and high amplitude waves at $25 \mathrm{~ms}$ and later made the identification of the postcentral gyrus possible nevertheless. Electrical mapping of the motor cortex took up to 30 minutes until a clear result was obtained. It was successful in 37 patients, but failed in three patients with precentral and central lesions. Functional neuronavigation indicating the tumour margins and the motor and sensory evoked fields was possible in all patients.

Conclusion: The SEP phase reversal of N20-P20 is a simple and reliable technique, but the success rate is much lower in large central and postcentral tumours. With the use of polyphasic late waveforms the sensorimotor cortex may be localised. By contrast with motor electrical mapping it is less time consuming. Functional neuronavigation is a desirable tool for both preoperative surgical planning and intraoperative use during surgery on perirolandic tumours, but compensation for brain shift, accuracy, and cost effectiveness are still a matter for discussion.
\end{abstract}

A ccurate preoperative and intraoperative localisation of the sensorimotor cortex is an essential adjunct to successful surgical excision of tumours involving the precentral and postcentral gyri. During the past decade the use of anatomical landmarks usually displayed by $\mathrm{CT}^{1}$ or $\mathrm{MRI}^{2}$ has been further developed by advanced functional neuroimaging techniques which include functional MRI (fMRI), ${ }^{3-6}$ magnetic source imaging (MSI), ${ }^{78}$ and PET. ${ }^{9}$ These non-invasive diagnostic tools not only provide excellent information for presurgical planning but their integration into neuronavigational systems allows safer and more radical surgery in and around the sensorimotor cortex. ${ }^{4810}$

Traditionally, intraoperative identification of the perirolandic gyri has been accomplished using electrophysiological techniques, either electrical stimulation mapping of exposed motor cortex ${ }^{11-13}$ or recording the phase reversal of somatosensory evoked potentials (SEPs) across the central sulcus. ${ }^{114-20}$ So far they have not become obsolete because the availability of functional neuronavigation is still limited, methodological problems may complicate the recording of fMRI or MSI in some cases, and its cost effectiveness is a matter for discussion. ${ }^{21} 22$

The recording of SEP phase reversal is considered a reliable tool and has been regarded as a reference standard for functional neuroimaging studies ${ }^{1023}$; however, several studies reported on limitations and failures of the technique especially in patients undergoing surgery for removal of perirolandic mass lesions. ${ }^{1152425}$ A high variability of SEP latencies and amplitudes is found in the presence of a tumour, ${ }^{11}{ }^{14}$ but detailed analyses of cortically recorded SEP waveforms were mainly performed in non-lesional patients with epilepsy ${ }^{192627}$ or were based on case reports. ${ }^{142425}$ The purpose of this study was to examine the feasibility and the waveform characteristics of SEP phase reversal under the prerequisites of space occupying perirolandic tumours varying in size, anatomical location, and functional sensorimotor impairment in a large consecutive series. In two subgroups the benefit from combinations with electrical motor cortex stimulation and functional neuronavigation was examined.

\section{MATERIALS AND METHODS \\ Patients}

In 230 consecutive patients undergoing open surgery the localisation of the central sulcus was performed by SEP recordings from the exposed cortex. Their mean age was 46.5 years (SD17.7) including five children between the ages of 6 and 14. Eleven patients were examined twice because they required a second operation on a recurrent tumour.

In all patients space occupying mass lesions in and around the sensorimotor cortex were diagnosed by preoperative CT or

Abbreviations: SEP, somatosensory evoked potential; MSI, magnetic source imaging; CS, central sulcus; MEF, motor evoked magnetic field 
Table 1 Distribution of diagnoses in 230 patients

\begin{tabular}{lll}
\hline Diagnosis & $\mathrm{n}$ & $\%$ \\
\hline Glioma: & & \\
$\quad$ Grade I, II (WHO) & 38 & 17 \\
Anaplastic astrocytoma & 14 & 6 \\
Oligodendroglioma & 11 & 5 \\
Glioblastoma & 47 & 20 \\
Other gliomas & 12 & 5 \\
Metastasis & 41 & 18 \\
Meningioma & 37 & 16 \\
Other & 30 & 13 \\
Patients & & \\
\hline
\end{tabular}

MRI, the average maximum tumour size was $3.8 \mathrm{~cm}$ (SD 1.2) in diameter. Patients undergoing surgery for epilepsy without structural brain lesions and arteriovenous malformations were excluded. Glioma was the most frequent diagnosis, followed by metastases, meningiomas, and other tumours (table 1). Seventy two patients (31\%) showed focal sensorimotor deficits preoperatively (44 mono or hemiparesis, 12 hypaesthesia, 16 both); 158 patients were asymptomatic before surgery $(69 \%)$; they were diagnosed after transient or non-specific clinical symptoms such as convulsions or headache (table 2).

Only space occupying lesions within or adjacent to the precentral and postcentral gyri were included in the study. The patients were divided into five groups (fig $1 \mathrm{~A}$ ): Frontal lesions adjacent to $(\mathrm{a} ; \mathrm{n}=41)$ or invading $(\mathrm{b} ; \mathrm{n}=76)$ the precentral gyrus, central tumours below or within the rolandic fissure (c; $n=49)$, and parietal lesions invading $(d ; n=39)$ or adjacent to the postcentral gyrus $(\mathrm{e} ; \mathrm{n}=25)$. Patients harbouring extra-axial tumours such as meningiomas were assigned to a group according to that area on the brain surface where the maximum compression of cortical gyri was found. The classification and clinical data are summarised in figure 1 and table 2.

\section{METHODS}

All patients were operated on under general anaesthesia using midazolam, nitrous oxide, and a short acting muscle relaxant (succinylcholine, atracurium besylate) for induction. During surgery muscle relaxants were avoided and anaesthesia was maintained with intravenous infusion of propofol and fentanyl, sufentanil, or alfentanil. Immediately after the operation the surgeon was asked to give his estimation of the completeness of tumour removal (complete, subtotal, or biopsy). All patients were examined by CT or MRI between 1 day and 3 months after surgery, but this information is not included in this study.
The SEPs were recorded in a four or eight channel montage using a Pathfinder I or a Viking IV-P (Nicolet Instruments, Madison, WI, USA). Display and nomenclature of SEP peaks, as well as stimulation and recording variables, were chosen according to commonly used standards. ${ }^{28}$ The median nerve contralateral to the lesion was stimulated at the wrist with 5.1 pulses/second and a current intensity between $5 \mathrm{~mA}$ and 10 $\mathrm{mA}$ until slight twitches of the thumb were obtained. A subdermal needle electrode at the forehead served as the reference (length $12 \mathrm{~mm}, 26$ gauge), all recordings were performed using a filter band pass of $30-3000 \mathrm{~Hz}$ and a time base of $50 \mathrm{~ms}$. After dura opening the surgeon was asked where he would expect the central sulcus to be located, if he could recognise the tumour mass on the cortical surface, and if the perirolandic gyri were displaced or infiltrated by the tumour. In 80 patients additional information was obtained from functional neuronavigation (see below).

In 154 patients a grid and in 76 patients a strip electrode with $10 \mathrm{~mm}$ interelectrode spacing (Cortac, $5 \times 4$ contact grid or 4,6 , or 8 contact strip electrode, PMT Corporation, Chanhassen, MN, USA) was placed over the putative central sulcus. The waveforms recorded from the 20 contact array were obtained by sequentially recording five channels at a time using a switchboard. Although grid electrodes were preferred generally strips were chosen in case of small craniotomies ( $<5 \mathrm{~cm}$ in diameter), in patients operated on for meningiomas or recurrent tumours and if bridging veins prevented safe placement on the cortical surface. The placement of the electrode was intended (1) to cross the putative central sulcus, (2) to cover the hand area of the sensorimotor gyri, (3) to make an angle of 15 degrees with the sagittal direction, and (4) not to cover the centre of the lesion but rather to lie adjacent to the visible margins of the tumour mass. The location of the electrode was adjusted to obtain maximum peak amplitudes by moving it in a mediolateral and frontoparietal direction or rotating it by angles of 15 degrees (fig $1 \mathrm{~B}$ ).

In addition the hand area of the precentral gyrus was localised using electrical stimulation in 40 patients. The mapping technique we used for cortical stimulation is similar to that reported by others during both cerebral tumour and epilepsy surgery. ${ }^{16} 172029$ Two neighbouring contacts of the grid electrode were used to apply a maximum of 250 constant current rectangular pulses at a rate of $50.1 \mathrm{~Hz}$ and a duration of $0.1 \mathrm{~ms}$. The stimulus intensity was adjusted gradually by increments of $2 \mathrm{~mA}$ each to a maximum of $30 \mathrm{~mA}$ until a tonic movement of the contralateral hand was produced. With the onset of the stimulation effect the train was discontinued and the current intensity was written down. The motor hand area was determined at the location where the lowest current intensity produced movement effects of the hand.

Eighty operations were planned and performed with image guided frameless stereotaxy in combination with MSI. After sensory and motor activation the localisation of magnetoencephalographic sources on the basis of a single current dipole

\begin{tabular}{|c|c|c|c|c|c|c|c|c|c|c|}
\hline \multirow[b]{2}{*}{ Group } & \multicolumn{4}{|c|}{ Neurological deficit } & \multicolumn{3}{|c|}{ SEP localisation } & \multicolumn{3}{|c|}{$\begin{array}{l}\text { Electrical stimulation } \\
\text { mapping }\end{array}$} \\
\hline & None & Motor & Sensory & Both & Yes & No & Total & Yes & No & Tota \\
\hline A & 35 & 6 & 0 & 0 & 40 & 1 & 51 & 8 & 0 & 8 \\
\hline B & 66 & 9 & 0 & 1 & 73 & 3 & 76 & 7 & 2 & 9 \\
\hline C & 10 & 27 & 3 & 9 & 42 & 7 & 49 & 6 & 1 & 7 \\
\hline D & 27 & 2 & 6 & 4 & 33 & 6 & 39 & 9 & 0 & 9 \\
\hline $\mathrm{E}$ & 20 & 0 & 3 & 2 & 23 & 2 & 25 & 7 & 0 & 7 \\
\hline Patients (n) & 158 & 44 & 12 & 16 & 211 & 19 & 230 & 37 & 3 & 40 \\
\hline
\end{tabular}


A

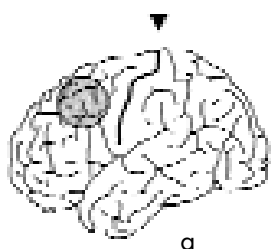

B

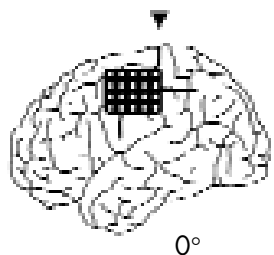

$0^{\circ}$

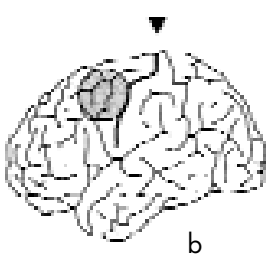

b

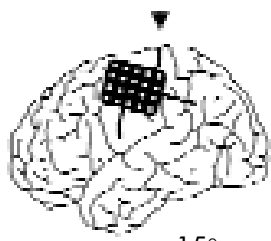

$15^{\circ}$

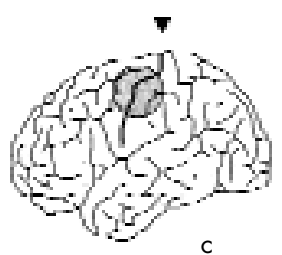

C

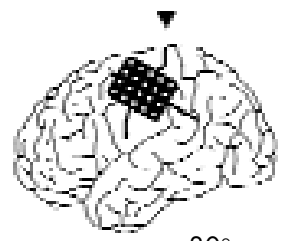

$30^{\circ}$

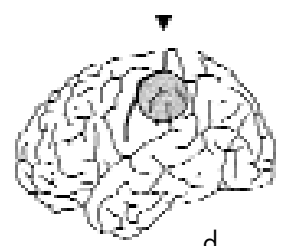

$d$

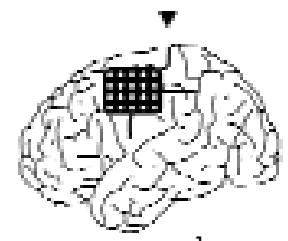

$-1 \mathrm{~cm}$

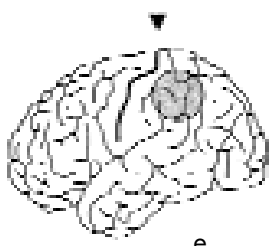

e

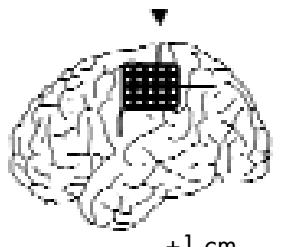

$+1 \mathrm{~cm}$
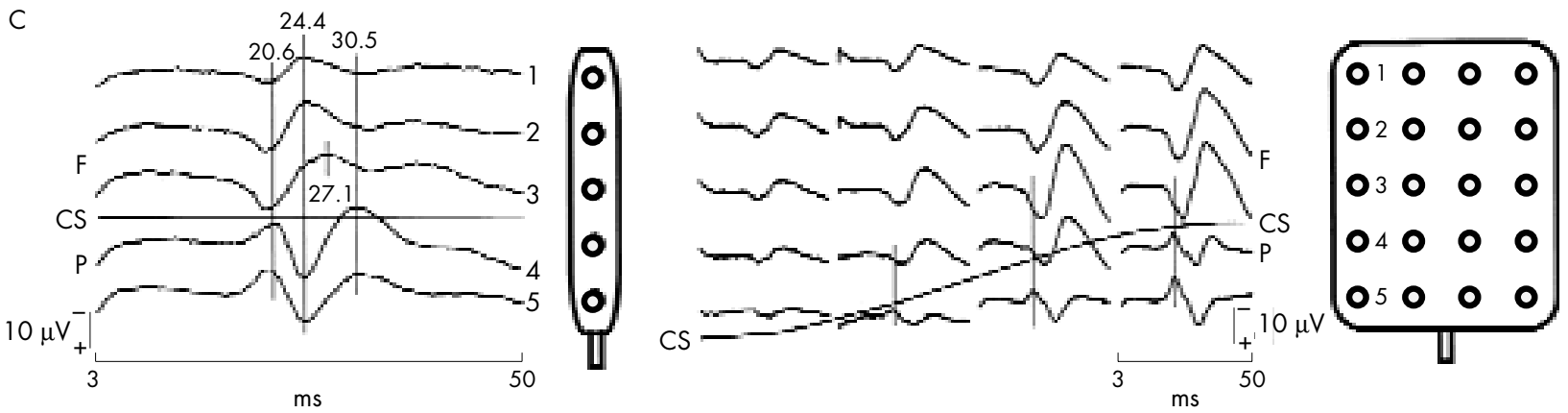

Figure 1 (A) Classification of patients into groups a-e according to tumour location in front of, within, and behind the perirolandic gyri. (B) The recording electrode was rotated and shifted sagittally across the central sulcus to obtain maximum amplitudes. (C) A typical phase reversal of the first cortical wave is shown obtained with a strip (left) and a grid (right) electrode. The course of the central sulcus (CS) is indicated by a line and a triangle. $F$, frontal; $P$, parietal.

model was performed preoperatively using the MagnesII biomagnetometer (Biomagnetic Technologies Inc, San Diego, CA, USA) and data were fused with the MRI serving for the intraoperative neuronavigation system. The Stealth Station (Surgical Navigation Technologies Inc, Broomfield, CO, USA) was used in 25 patients and the MKM neuronavigation system (Zeiss, Oberkochen, Germany) in 55 patients. Our technique of image guided frameless stereotaxy combined with magnetoencephalography has been published..$^{10} 30$

\section{RESULTS}

\section{Feasibility of SEP phase reversal}

The intraoperative localisation of the central sulcus with cortically recorded SEP was successful in 211 patients $(92 \%$; table 2 ). It was possible in all of the patients to put the electrode on the cortical surface with at least one contact behind or in front of the central sulcus; however, in seven patients the electrode had to be pushed subdurally beyond the margins of the craniotomy and the central sulcus was identified outside the area of dura opening. Great care was taken not to injure cortical bridging veins; in three patients this was the cause for changing from a grid electrode to a strip with four leads. Nevertheless, the rolandic fissure could be determined safely in these patients, too. The time spent on identifying the central fissure after dura opening usually did not exceed 5 minutes. No unwanted side effects were seen postsurgically except in two patients who developed meningitis within the first postoperative week; however, a clear connection between the inflammation and the SEP recordings could not be demonstrated.

In most cases cortically recorded SEPs were obtained after 50 to 100 averages, in a few patients only the number of sweeps was greater than 200. Interpeak amplitudes between the initial cortical and the subsequent waves between 25 and $45 \mathrm{~ms}$ were found in a wide range from $5 \mu \mathrm{V}$ to $120 \mu \mathrm{V}$, the latency of the first postcentral negativity, the N20 peak, was measured between $16.2 \mathrm{~ms}$ and $24.6 \mathrm{~ms}$ with a mean value of $21.6 \mathrm{~ms}$ irrespective of body size, age, temperature, and neurological deficits.

Intraoperative localisation of the central sulcus based on the recording of the inversion of a postcentral negative and a precentral positive peak between approximately $18 \mathrm{~ms}$ and 24 $\mathrm{ms}$ - the N20-P20 phase reversal-was possible in 188 patients $(82 \%)$ (figs $1 \mathrm{C}$ and $2 \mathrm{~A}$ ). In 23 patients ( $10 \%)$ the typical phase inversion at $20 \mathrm{~ms}$ was questionable or missing, but from the electrode lying over the postcentral gyrus characteristic late waveform components could be recorded: Either one single negative wave with a markedly high amplitude at approximately $35 \mathrm{~ms}$ in seven patients (fig $2 \mathrm{E}$ ) or a polyphasic sequence of positive and negative peaks between 25 and $45 \mathrm{~ms}$ in 13 patients (fig $2 \mathrm{~F}$ ) made the localisation of the postcentral gyrus possible. The recording of a cortical potential inversion failed and the localisation of the central sulcus was impossible in 19 patients ( $8 \%$; fig $2 \mathrm{D}$ ). During four operations major technical problems were encountered as could easily be recognised by the artifacts recorded. In two of them this resulted from loose cable connections, in one patient an inappropriate amplifier setup was chosen by mistake, and in one patient the reason remained unclear. In 15 patients the SEP waveforms remained flat or showed a large negative peak between 20 and $35 \mathrm{~ms}$ only although the recording was tried from various electrode sites (fig $2 \mathrm{D}$ ). The attempt to obtain clear waveforms was futile even after the electrode was shifted in an anterior-posterior or medial-lateral direction or after it was rotated by angles of 15 and 30 degrees. 


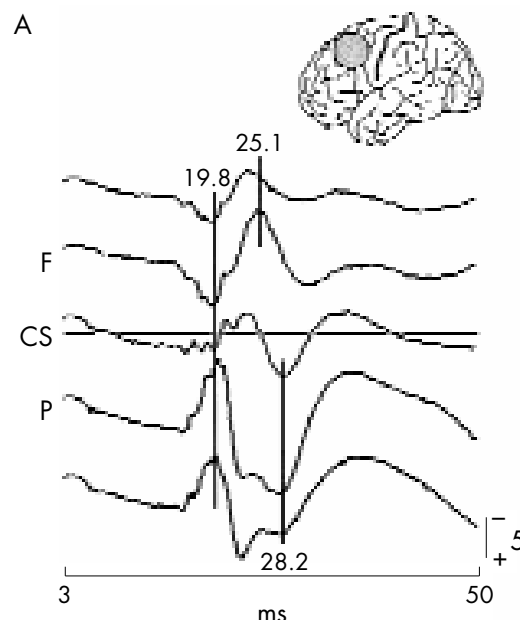

B
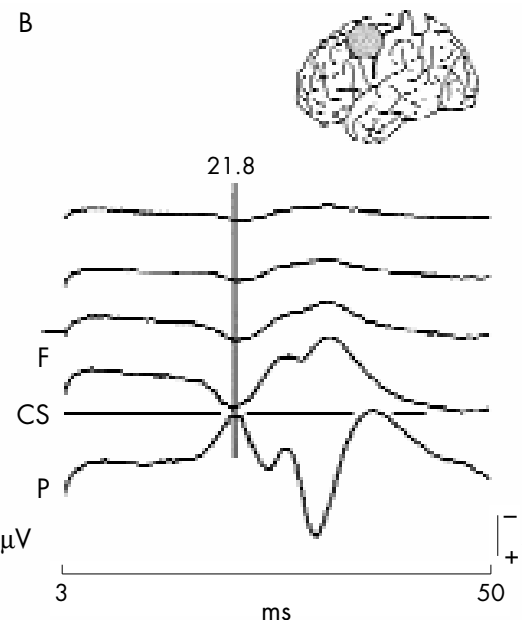

C

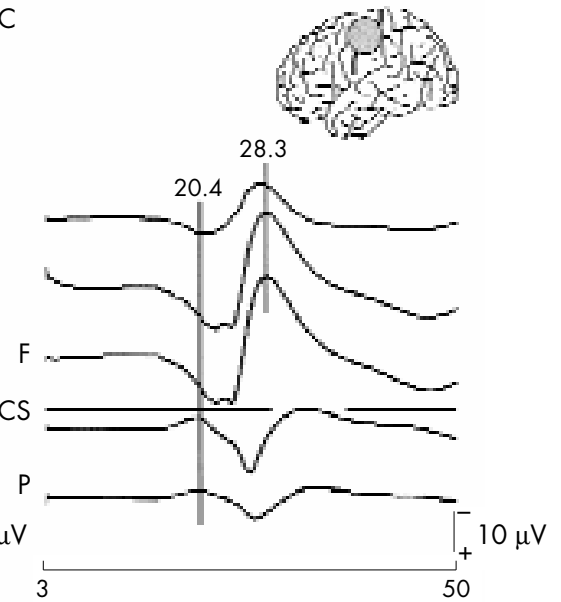

ms

F

E
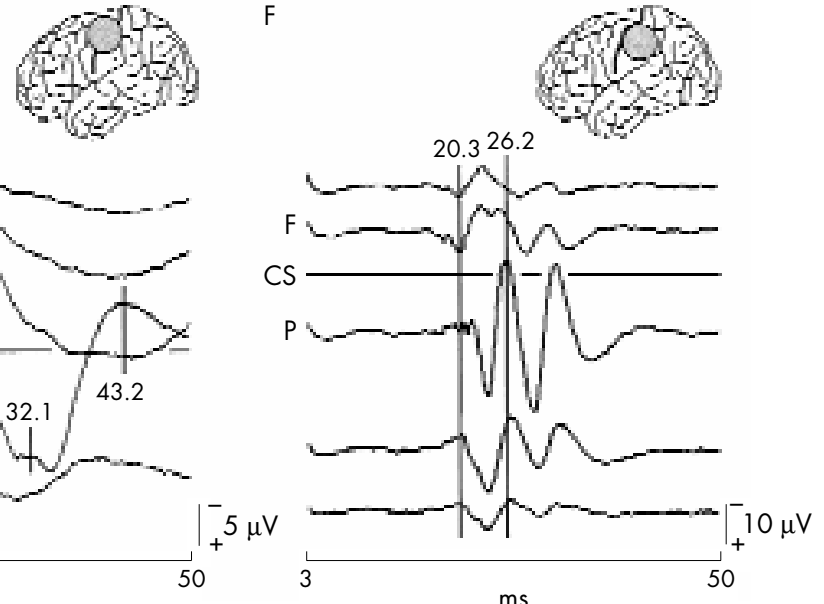

Figure 2 Intraoperative SEP recordings across the central sulcus (CS). The typical N20-P20 phase reversal shows (A) a transitional waveform recorded directly over the central sulcus. (B) Especially, in large precentral and central lesions some reduction of amplitudes was seen in frontal recordings, or (C) the N20-P20 waves were broadened and deformed, although a clear phase reversal was obtained. (D) The SEP phase inversion could not be recorded in 19 patients who showed no or extremely flat waveforms. In 23 patients the N20-P20 phase reversal was questionable or missing, but the postcentral gyrus was indicated either by (E) a large P25-N35 amplitude or (F) by a polyphasic configuration of waves at $25 \mathrm{~ms}$ and later. $F$, frontal; P, parietal.

\section{Feasibility of functional neuronavigation with MSI and electrical motor cortex stimulation}

During 80 operations both the SEP phase reversal and functional neuronavigation with MSI of the sensory and motor dipoles were performed (in part results from a subgroup of 50 patients published previously $\left.{ }^{10}\right)$. In all patients magnetoencephalographic localisation of the sensory and motor gyri was correct. This was confirmed by the SEP phase reversal in 77 patients (96\%). In two patients with large cystic gliomas (group B) the magnetoencephalographic localisations of the motor and sensory cortex were found adjacent to the tumour margins but no SEP phase reversal was obtained. During one operation SEP recordings were impossible because of defective cables.

The use of electrical stimulation mapping to localise the precentral gyrus was successful in 37 patients (93\%) (table 2; fig 3). The stimulation intensity necessary for movement effects of the contralateral hand or fingers ranged from $2 \mathrm{~mA}$ to $16 \mathrm{~mA}$. Higher current intensities up to $30 \mathrm{~mA}$ were necessary especially in patients presenting with a marked motor deficit or harbouring large cystic tumours. In three patients a further increase of stimulus intensity up to the maximum of $30 \mathrm{~mA}$ was futile at any cortical stimulation site. Six patients showed a motor movement of the contralateral limb evoked by stimulation at one circumscribed cortical spot only, but not from elsewhere. In 31 patients movement effects were elicited after stimulation at various sites on the brain surface (including the postcentral gyrus in five patients). In all of them the hand area of the motor cortex was determined according to the stimulation trial with the lowest stimulus intensity necessary for contractions of the hand. In two patients electrical stimulations were stopped immediately after the onset of jacksonian seizures. Both patients had a history of repeated convulsions, the intraoperative seizures were terminated promptly after a bolus of thiopental, midazolam, and atracurium besylate was given intravenously. No additional postoperative motor deficits or other adverse side effects were found in the two patients who developed focal motor convulsions after cortical stimulations. The time required for electrical stimulation mapping varied between 15 and 30 minutes and depended markedly on the maximum stimulus intensity of each pair of electrodes necessary to produce a movement effect. In three patients the motor area could not be identified with electrical cortex stimulation. One of them presented with severe paresis of the right arm and hemihypaesthesia preoperatively due to an astrocytoma grade 3 (World Health Organisation (WHO) ) symmetrically involving the central gyri (group C). Likewise no SEP phase reversal could be obtained 
A

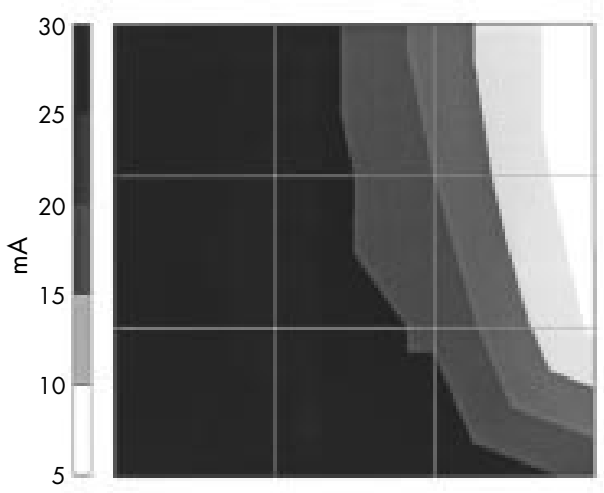

B

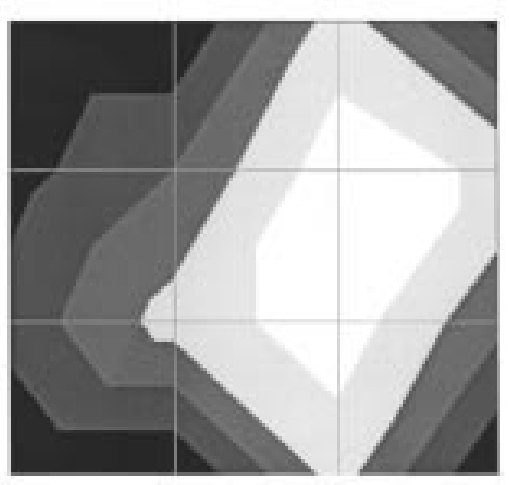

C

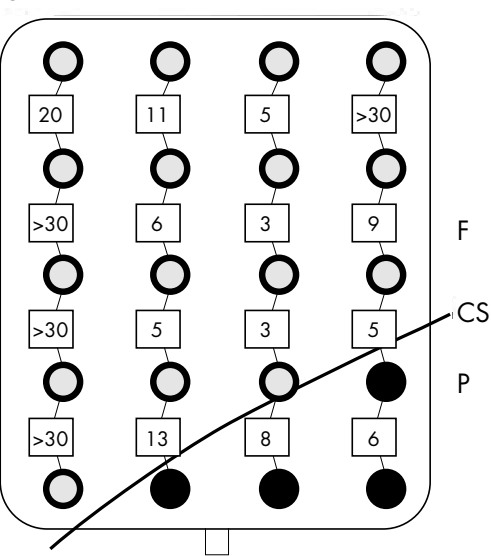

Figure 3 Electrical motor cortex stimulation. The hand area of the precentral gyrus is determined after serial mapping of the cortex underneath the grid electrode. (C) For each stimulation trial the numbers interconnecting two electrode contacts give the lowest current intensity in $\mathrm{mA}$ necessary to elicit a motor response. (A) Two dimensional contour plots show the motor hand area (bright area) close to the margin of the electrode and, $(B)$ after correcting its position, near the centre of the grid. Stimulation effects were obtained with the minimum current intensity at the precentral motor hand area $(3 \mathrm{~mA})$, but motor responses after postcentral stimulation were also possible. CS, central sulcus; $F$, frontal; $P$, parietal.

in this patient and a biopsy only was taken from the tumour. Two patients were operated on for large mainly cystic glioblastomas extending up to the cortical surface (group B), on admission both showed mild hemiparesis. During surgery the brain was markedly swollen, after mannitol was given a SEP phase reversal was obtained but electrical stimulation failed. Only the anterior parts of the tumour masses were resected and no postoperative neurological worsening was seen.

\section{Waveform characteristics of SEP recorded from the exposed cortex}

The effect of a mass lesion (groups a and e) on the SEP In patients with tumours located distant from the central sulcus (groups a and e) SEP recordings from the postcentral cortex and 2 or $3 \mathrm{~cm}$ behind showed the well known sequence of peaks N20, P25, and N35. This peak configuration was similar to that recorded from the scalp at C3' or C4' with amplitudes being at least five times higher. By contrast with the postcentral N20 the positive wave P20 was found constantly in recordings from the precentral gyrus and in front of it. Waveforms were more complex if the electrode was lying adjacent to or directly over the central sulcus: A varying number of small peaks inserted into the major waves was seen giving the impression of a transitional and less smooth waveform (fig 2 A). Potentials at about $20 \mathrm{~ms}$ equidistantly recorded from behind and in front of the central sulcus did not show exact peak inversion, especially the frontal positive mirror image to the postcentrally recorded N20 exhibited a considerable variety of peak latencies. The expected precentral wave P20 was found with both an increase and a decrease in latency of up to $2 \mathrm{~ms}$; however, this was not an obstacle to clear localisation of the central sulcus.

The most prominent succeeding components were a postcentral negative wave between 30 and $40 \mathrm{~ms}$ (N35) and a precentral N27 in recordings adjacent to the central sulcus. At more posterior recording sites the pattern of the postcentral N35 was not changed except for a gradual reduction of amplitude. Regarding the precentral N27 a reduction in both amplitude and latency was often noted after having moved the electrode to more anterior sites-for example, from N27 to $\mathrm{N} 25$ or N24 with an interelectrode distance of $1.0 \mathrm{~cm}$ (fig l C, left). In general there was no systematic effect of the tumour masses in groups a and e on the SEP phase reversal despite the fact that some of the patients had motor or sensory deficits (table 2).
The effect of a mass lesion (groups b, c, and d) on the SEP

Patterns of SEP waveforms in patients belonging to group $b$ were similar to those recorded in patients in groups a and e, but in most cases the precentral peaks were found considerably smaller in amplitude compared with the peaks recorded from behind the central sulcus. Precentrally recorded amplitudes of P20 and N27 were found the smaller the larger the underlying tumour mass was (fig $2 \mathrm{~B}$ ). In nine patients belonging to group $\mathrm{b}$ a motor deficit was seen preoperatively, but there was no noticeable systematic effect of the paresis on the SEP waveforms.

Eighty per cent of the patients with lesions that affected both the precentral and the postcentral gyrus (group C) exhibited preoperative neurological deficits (table 2). Although it was necessary in almost all patients to place at least some leads of the electrode across the tumour surface, in this group a phase reversal N20-P20 was also obtained, but there was a considerably higher number of patients in whom the recording of a peak inversion was difficult. In almost all patients it was necessary to repeatedly move the electrode in an anterior-posterior and a medial-lateral direction or to rotate it by angles of 15 or 30 degrees until the presumed central sulcus was clearly identified.

In patients belonging to groups $\mathrm{c}$ and d two distinct SEP patterns were seen. Especially if the postcentral cortical surface was flattened by the underlying space occupying tumour mass, either a prominent wave P25-N35 or a polyphasic sequence of peaks beginning at approximately $25 \mathrm{~ms}$ could be recorded (fig $2 \mathrm{E}$ and $\mathrm{F}$ ). The polyphasic waveform patterns showed three or four single peaks of similar amplitude and appeared different from the usual V or W shaped N20-P25N35 sequence. Although these variable late components were recordable from several prerolandic and postrolandic sites the tracing picked up from immediately posterior to the central sulcus was markedly higher in amplitude and thus served to identify the postcentral gyrus. This was found with and without N20-P20 phase reversal, but the peak N20 was usually somewhat flattened and prolonged.

\section{Clinical results}

Without considering tumour size, location, histological diagnosis, grading, and preoperative neurological deficits the surgeon's assessment of the extent of tumour removal was "complete" in 93, "subtotal" in 121, and "biopsy only" in 16 patients 
Table 3 Postoperative clinical results

\begin{tabular}{llllll}
\hline Tumour removal & $\mathrm{n}$ & $\%$ & Postoperative neurological findings & $\mathrm{n}$ & $\%$ \\
\hline Complete & 93 & 40.4 & Same or better & 192 & 83.5 \\
Subtotal & 121 & 52.6 & Worse & 38 & 16.5 \\
Biopsy & 16 & 7.0 & Transient mild paresis & 28 & 3 \\
\multirow{2}{*}{ Patients } & \multirow{2}{*}{100} & Transient aphasia & Transient severe paresis & 3 \\
& 230 & \multirow{2}{*}{} & & Prolonged severe paresis & 4 \\
\hline
\end{tabular}

(table 3). The postoperative sensorimotor function was unchanged or improved in 192 patients $(83.5 \%)$. Twenty eight patients demonstrated transient mild hemiparesis postoperatively, but regained a normal sensorimotor status within 1 week. Transient aphasia was noticed in three patients $(1.3 \%)$, one with an astrocytoma grade 3 (WHO) and two with large glioblastomas extending from the postcentral parietal cortex to the posterior temporal lobe. Seven patients $(3.0 \%)$ showed severe focal motor deficits immediately after surgery. In three of them the hemiparesis improved gradually within 12 days and they were able to use this limb in daily life (paresis grade $4 / 5)$. In four patients a severe monoparesis of the contralateral arm was found lasting longer than 2 weeks. Postoperative CT showed marked local brain oedema around the resection area in two patients; in one of them it was soaked with blood. The other two patients (group b) with severe paresis had an oligodendroglioma and a recurrent astrocytoma grade 3 (WHO); the postoperative CT suggested that the descending motor pathways might have been affected surgically.

In the group of 80 patients undergoing surgery with functional neuronavigation and SEP recordings 77 (96.3\%) exhibited unchanged or improved sensorimotor function postoperatively, but two patients (2.5\%) developed mild transient motor weakness (one low grade astrocytoma in group b and one astrocytoma grade 3 in group A) and one had a marked but incomplete hemiparesis postoperatively (glioblastoma in group a).

\section{Illustrative case}

Shifting and rotating the electrode on the exposed somatomotor cortex was particularly important in patients who did not show the above mentioned waveform criteria for identification of the rolandic fissure after the first recording was done. A 32 year old woman presented with weakness of the right arm and mild dysphasia. Brain MRI showed a left sided tumour with positive contrast enhancement presumably within the central region. A small craniotomy was centred onto the tumour contour which was provided by the neuronavigational microscope (MKM system, Zeiss, Oberkochen, Germany) and a four contact strip electrode was placed over the putative central sulcus adjacent to the tumour margin (fig 4, left). The first SEP recording showed a negative wave between 21 and $22 \mathrm{~ms}$ in all four tracings, but no phase reversal and no polyphasic late components (fig $4 \mathrm{~A}$ ). As the strip electrode was crossing the tumour contour, which was displayed by the neuronavigational microscope, the second recording was performed after having the electrode rotated by 30 degrees to move the strip away from the tumour surface and to cover the suspected hand area of the postcentral gyrus. The recordings now showed an increasing amplitude of the negative peaks in the two posterior recordings and a decreasing amplitude in the two anterior recordings, but yet there was no phase reversal (fig $4 \mathrm{~B}$ ). Finally the electrode was shifted more rostrally and it was rotated by 15 degrees to cross the gyri in a perpendicular alignment. Now a clear phase reversal
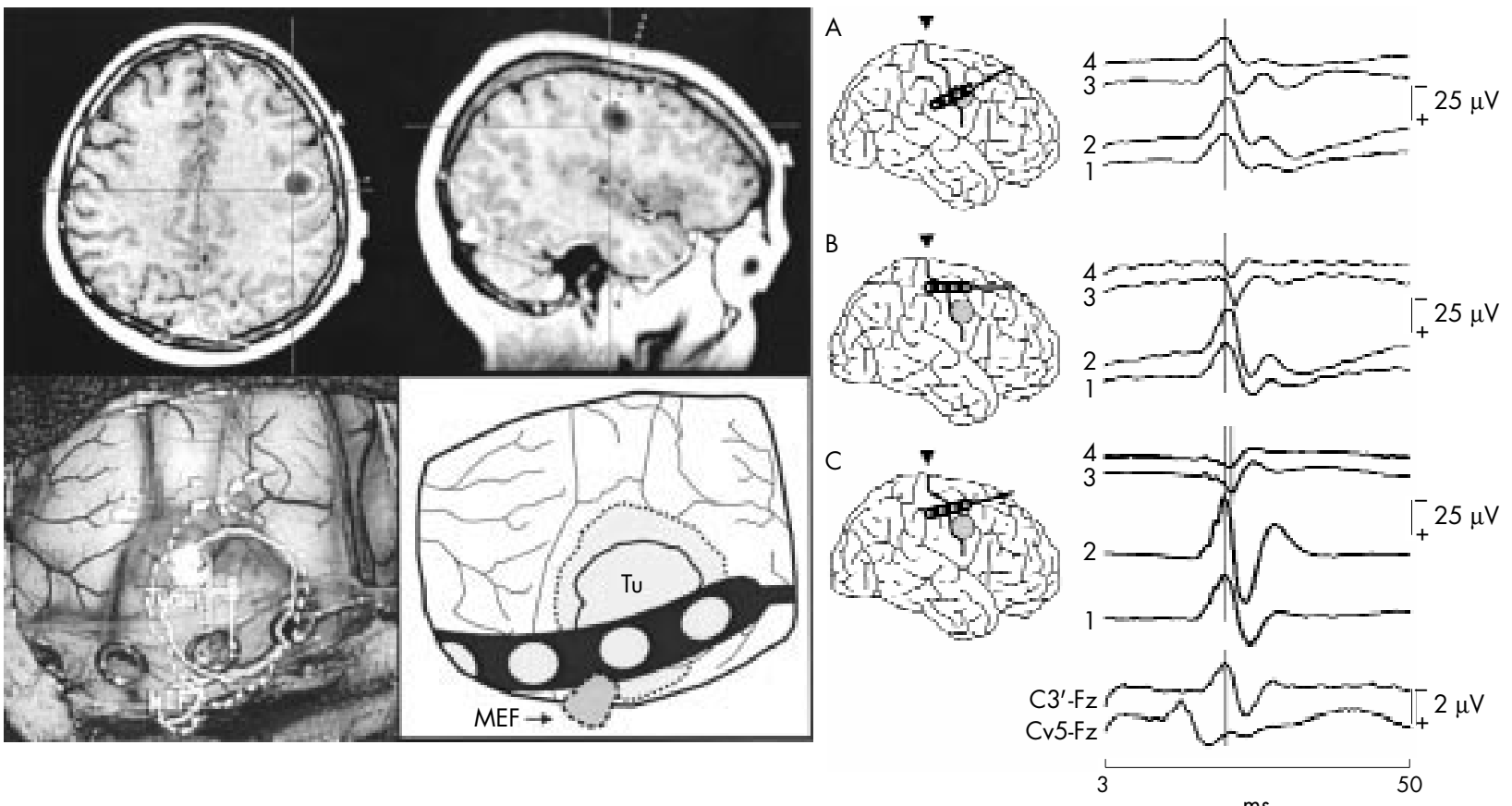

$\mathrm{ms}$

Figure 4 Illustrative case. The MRI suggested a tumour of the central region, the intraoperative aspect showed a broadened gyrus enclosing a superficial lesion (Tu) as delineated by the neuronavigational miscroscope (dotted line). The motor magnetoencephalographic source (MEF) was found in the anterior bank of this gyrus. The N20-P20 phase reversal was obtained after shifting and rotating the electrode away from the tumour centre (A, B, and C). Refer to text for details. 
was obtained between a large N20 at $21.4 \mathrm{~ms}$ (curve 2) and a small positive wave at $22.2 \mathrm{~ms}$ (curve 3 ) making the identification of the central sulcus possible (fig $4 \mathrm{C}$ ). The first electrode (curve 1) was situated $1 \mathrm{~cm}$ more posteriorly and showed a decrease of amplitudes, but no change in latencies. The dipole of the motor evoked magnetic field (MEF) was found in the anterior bank, but not in the centre of the precentral gyrus adjacent to and slightly dislocated by the tumour. The intraoperative pathohistological finding suggested a glioblastoma and subtotal removal was performed only. After surgery the patient showed slight improvement of her motor weakness and dysphasia.

\section{DISCUSSION}

The SEP phase reversal technique is routinely used during both surgery on tumours and non-lesional epilepsy surgery around the sensorimotor cortex. The principle is based on the polarity inversion of the primary cortical wave across the central sulcus provided that at least one recording site is located either behind or in front of it. In most cases it is possible to obtain a SEP phase reversal and to localise the rolandic fissure within a few minutes. ${ }^{11}{ }^{15-17} 192031-33$ If the cortical surface anatomy, the location of the tumour, or neuronavigational devices do not provide an idea of where the central gyrus is located a large grid or a long strip electrode with six leads or more is preferred to make sure that it is crossing the sensorimotor gyri. ${ }^{18}$ Rarely, this may be complicated by the small size of the craniotomy, cortical veins, or scars. During 80 operations performed with the combined use of SEP and neuronavigation it was very helpful to obtain additional information about the patients' individual anatomy and the correct placement of the electrode was made easier. The situation, however, is more complicated with an increase in tumour size, with severe preoperative neurological deficits and with lesions invading the precentral and postcentral gyri (groups b, c, and d). Under such circumstances reduced feasibility and reliability of the SEP phase reversal technique must be expected.

Gregorie and Goldring ${ }^{16}$ reported on SEP phase reversal and electrical motor cortex stimulation in 32 patients with tumours of the sensorimotor region. They presented illustrative case reports and demonstrated the effect of displacement and invasive growth of a tumour mass on the sulcal anatomy in the sensorimotor area. In some patients they could not obtain a phase reversal, but the cause remained unclear. Cedzich et $a l^{11}$ found a loss of N20 or P20 in 9\% of their patients and the recording of a phase reversal failed. They explained this finding by an "off axis" recording from electrodes that were not exactly aligned over the sensory and motor hand areas. Similarly, this phenomenon was seen by Wood et al, ${ }^{19}$ who recorded the SEP with independently positioned electrodes from a widely exposed sensorimotor cortex. They emphasised that "off axis" alignment of electrodes may not only make the recording of a phase reversal impossible, but may even provide misleading results during localisation of the central sulcus. By contrast with Cedzich et al their study was performed in patients with epilepsy without displacement or infiltration of the central gyri by a tumour mass. Aiba and Seki ${ }^{14}$ were unable to record a phase reversal in three of 18 patients due to severe preoperative motor deficits. Babu and Chandy ${ }^{15}$ found after 65 central operations for tumours that in patients with predominant motor disturbance the SEP phase reversal could be recorded in all patients, sensory deficits resulted in a reduction of amplitudes, and in six patients with marked sensorimotor deficits the attempt to record a SEP was futile. By contrast Sonoo $e^{2} \mathrm{al}^{24}$ reported on two patients with major sensory deficits and localised lesions of the postcentral gyrus in whom the scalp recording of $\mathrm{N} 20-\mathrm{P} 20$ and later waveforms were eliminated and only a widespread frontal negativity was obtained. Surprisingly, in a patient with a precentral astrocy- toma Suzuki and Yasui ${ }^{25}$ found a clear SEP phase reversal not only across the central sulcus but also across the precentral sulcus.

These examples show that perplexing findings must be expected occasionally in patients with tumours: In a large operative series of lesions differing in size, growth pattern, location, and functional impairment about $10 \%$ of the patients will not show the classic N20-P20 inversion, possibly as a result of three causes: (1) the tumour desynchronises the propagated afferent electrical volleys along the thalamocortical pathways, (2) the mass effect of the lesion distorts the spatiotemporal projection of cortical electrical dipoles to the brain surface, and (3) the recording site may not be appropriate for recording a potential generated in the hand area of the postcentral gyrus. In our series of 230 patients with tumours the location of the lesion clearly showed an effect on the rate of successful SEP recordings. It is not surprising that the impact was greater in patients with lesions within the precentral or postcentral gyri (groups b, c, and d) by contrast with lesions in front or behind them (groups a and e). As expected, the incidence of neurological deficits was highest in patients belonging to group c; however, the rate of successfully recorded SEPs was almost identical in groups $\mathrm{c}$ and $\mathrm{d}$. This suggests that the space occupying effect of a tumour may be more important than sensorimotor deficits. We did not prove this statistically because of the great variability in tumour size, growth pattern, and location, but in many of our patients examined with both SEPs and neuronavigation the motor and sensory hand areas were found markedly compressed and displaced by the tumour (as indicated by the corresponding magnetic fields) although neurological symptoms were mild or even absent. Obviously an electrically silent lesion acts as a passive volume conductor and causes a spatiotemporal distortion of electrical dipoles projecting to the cortical surface and a loss of SEP waveforms without major clinical consequences.

In 20 patients we found the N20-P20 phase reversal being distorted considerably or lost, but the electrode located over the postcentral gyrus showed a typical polyphasic waveform between 25 and $45 \mathrm{~ms}$ that is not found in standard scalp recordings. This sequence of peaks was clearly distinct from a transitional waveform obtained from an electrode that lies directly over the central sulcus and records the spatiotemporal summation of the mirroring postcentral and precentral potentials. Wood et $a l^{19}$ suggested that the large positivenegative waveform at 25 and $35 \mathrm{~ms}$ may serve as an additional localising criterion, because it is usually recorded with the highest amplitude from the postcentral gyrus at a location 10 $\mathrm{mm}$ medial to the hand area. By contrast with Wood et al we saw a polyphasic sequence of waveforms beginning at $25 \mathrm{~ms}$, probably because of a difference in the filter settings. Wood et al used band pass filters of $1 \mathrm{~Hz}-1000 \mathrm{~Hz}$ and found that the postcentral P25 may easily interfere with the precentral P20-N30 phase reversal. In our recordings a $30 \mathrm{~Hz}-3000 \mathrm{~Hz}$ band pass filter was applied and the highest amplitude of the polyphasic sequence was recorded from the postcentral gyrus exclusively, thus serving as a safe localising criterion.

Electrical dipole generators contributing to the superficially recorded SEP waveforms have been examined thoroughly, and the number, the location and the orientation of neuronal sources contributing to the N20-P30 and P25-N35 waveform components have been discussed in detail..$^{19}{ }^{34-37}$ The most widely accepted models describe two single dipole sources separated spatiotemporally within the postcentral gyrus, which generate the N20-P30 and the P25-N35 waveform components. ${ }^{2637}$ To understand the cortical representation of the motor and somatosensory system studies have been performed using dipole source calculation methods, ${ }^{26} 3638$ electrical stimulation mapping of the sensorimotor areas in awake patients, ${ }^{31}{ }^{39-41}$ and functional imaging techniques (fMRI, MSI, PET). ${ }^{323} 4243$ Some of the results seem controversial; however, there is increasing evidence that the sensorimotor system 
must be understood as a complex and dynamic neuronal network. ${ }^{41}{ }^{44}$ Lehéricy et a $l^{45}$ combined fMRI data with electrical stimulation mapping and found a high correspondence between the motor somatotopical anatomy and function even in patients with tumours within the central region. Bittar et $a l^{23}$ used PET studies to demonstrate with statistical significance that central lesions were more often associated with altered activations, typically including secondary sensorimotor areas. In patients with tumours or angiomas of the sensorimotor cortex it has been shown that the reorganisation of both cortical somatosensory receptive fields and the motor efferent system is possible to some extent. ${ }^{6}{ }^{46-49}$ Plasticity of the brain is only one reason for the great interpatient variability of intraoperatively recorded SEP waveforms, drug effects during anaesthesia, neurological deficits and-most important of all-the influence of a tumour mass of variable size, location, and growth pattern make it extremely difficult to delineate systematic effects. In a heterogenous clinical series of patients with tumours it is desirable to rely on well known waveform patterns that may easily be recognised after the first SEP recordings have been obtained from the central region. One template is the classic N20-P20 phase reversal; in addition we could show that the recording of a polyphasic sequence of waves from the postcentral gyrus serves well as another localising criterion.

We have been able to localise the central sulcus with either SEP phase reversal or electrical motor cortex mapping in about $92 \%$ of the patients, in only one individual both techniques failed. This patient did not belong to those being operated on with functional neuronavigation, but because motor and sensory MSI was successful in all 80 patients examined $(100 \%)$, it seems very likely that the method could have helped in this special case, too. After operations exclusively performed with the help of SEP $83.5 \%$ of patients revealed unchanged or better clinical findings, whereas the percentage of patients without postoperative worsening was markedly higher $(96.3 \%)$ in those operated on with the additional use of functional neuronavigation. On the one hand this may result from the fact that functional imaging provides the opportunity to perform preoperative patient selection and meticulous surgical planning. ${ }^{50}$ According to the topographic relation between a tumour mass and the motor or sensory magnetoencephalographic sources the patients' risk for developing additional morbidity may be assessed as high or low. They will either be excluded from open surgical therapy or the extent of surgical resection will be adapted individually. Secondly, it is difficult to evaluate the feasibility of various localising techniques on the basis of postoperative sensorimotor findings as a criterion of assessment because patients may have benefited from the additional use of intraoperative frameless stereotaxy.

Functional imaging is a rapidly developing technique and its incorporation into neuronavigation is an important contribution to surgery in eloquent brain areas. Future applications will be the functional mapping of cortical areas associated with visual and language functions as well as imaging of fibre pathways connecting functional brain regions. Nevertheless some questions remain to be answered: (1) the localisation of the sensorimotor hand area by MSI compared with fMRI and PET has been reported to differ between 10 and $20 \mathrm{~mm},{ }^{42}$ (2) the deformation of the cortical surface during operation on a central tumour, known as brain shift, may range up to more than $20 \mathrm{~mm}$ and thus markedly reduces the reliability of functional neuronavigation..$^{51}$ (3) By contrast with the MSI technique fMRI is generally available on standard 1.5 Tesla MRI systems, but it shows an error rate of up to $20 \%$ for localisation of the central fissure in patients with brain tumours. ${ }^{52}$

The purpose of functional neuronavigation reaches far beyond the mere localisation of the central sulcus, but this one simple task can be fulfilled by the SEP phase reversal technique, which is extremely fast, reliable, and cost effective. Provided that a tumour mass has not distorted the patient's sulcal anatomy of the cortex the localisation of the central sulcus is most important for the surgeon to preserve the precentral and postcentral gyri. However, in patients with large lesions the physiological relation of functional areas to the sulcal anatomy may be deformed markedly. Under these conditions the SEP phase reversal method is unable to provide sufficient information about the individual somatotopic organisation of the precentral gyrus. This can be delineated accurately by electrical stimulation mapping of the hand, leg, and face area of the motor cortex, either in the awake patient ${ }^{12}$ or with the help of multichannel electromyographic recordings. ${ }^{13}$

If necessary, electrophysiological recordings and stimulations can be performed repeatedly during the ongoing operation, the electrical stimulation of superficial and descending motor pathways bears the potential for real time continuous monitoring. ${ }^{13554}$ As a result of different overall capabilities the cost effectiveness of functional neuronavigation and the SEP technique cannot be compared easily. ${ }^{21}$ The costs for the purchase and the maintenance of the equipment (the electrodiagnostic and the neuronavigational system, fMRI, or MSI) differ markedly, the costs/patient depend on the price of a single use cortical electrode on the one hand and on the price of preoperative diagnostic and functional MRI examinations on the other. As costs are a matter for discussion the surgeon should select the appropriate technique for each single case specifically, however, to further improve safety and effectiveness of surgery within the central region the complementary effects of several techniques are desirable. Especially in high risk patients a future perspective is offered by the combined use of electrophysiological identification of deep seated functional areas with three dimensional visualisation of corticospinal pathways ${ }^{55}$ and intraoperative MRI.

\section{CONCLUSION}

The cortical recording of the N20-P20 phase reversal of somatosensory evoked potentials is a simple and reliable technique to localise the central sulcus on the brain surface. It has gained widespread availability, the recording equipment is low priced and the costs/patient are restricted to the price of one electrode grid or strip. Although the success rate is rather high in general $(>90 \%)$, in large central and postcentral lesions the recording of a typical N20-P20 phase reversal may be questionable or even impossible.

Late waveform components of the median nerve SEP (a high negative wave or a polyphasic sequence of positive and negative peaks between 25 and $45 \mathrm{~ms}$ ) can serve as an additional index to identify the postcentral gyrus or the central sulcus respectively. Although the success rate of the SEP phase reversal can be improved by these indices, the method has drawbacks: (1) accurate localisation of the hand area of the motor cortex requires electrical stimulation mapping of the central region; to map the somatosensory cortex the patient needs to be awake during surgery. (2) The effect of large central tumours on the cortical SEP recorded after tibial nerve or trigeminal nerve stimulation has not yet been examined systematically and the feasibility of the SEP technique has not been verified for the leg and face area of the somatosensory cortex. (3) The SEP phase reversal is a method for localisation of the cortical surface only. During the removal of deep seated tumours within the white matter functional neuronavigation is superior because of its capability to offer three dimensional spatial information in combination with functional data.

\section{Authors' affiliations}

J Romstöck, R Fahlbusch, O Ganslandt, C Nimsky, C Strauss,

Department of Neurosurgery, University of Erlangen-Nuremberg, Erlangen, Germany 


\section{REFERENCES}

1 Ebeling $U$, Huber $P$, Reulen $H J$. Localization of the precentral gyrus in the computed tomogram and its clinical application. J Neurol 1986;233:73-6.

2 Berger MS, Cohen WA, Ojemann GA. Correlation of motor cortex brain mapping data with magnetic resonance imaging. J Neurosurg 1990;72:383-7.

3 Boakye M, Huckins SC, Szeverenyi NM, et al. Functional magnetic resonance imaging of somatosensory cortex activity produced by electrical stimulation of the median nerve or tactile stimulation of the index finger. J Neurosurg 2000;93:774-83.

4 Nimsky C, Ganslandt O, Kober H, et al. Integration of functional magnetic resonance imaging supported by magnetoencephalography in functional neuronavigation. Neurosurgery 1999;44:1249-55.

5 Puce A, Constable RT, Luby ML, et al. Functional magnetic resonance imaging of sensory and motor cortex: comparison with electrophysiological localization. J Neurosurg 1995:83:262-70.

6 Roux FE, Boulanouar K, Ibarrola D, et al. Functional MRI and intraoperative brain mapping to evaluate brain plasticity in patients with brain tumours and hemiparesis. J Neurol Neurosurg Psychiatry 2000;69:453-63.

7 Ganslandt O, Ulbricht D, Kober H, et al. SEF-MEG localization of somatosensory cortex as a method for presurgical assessment of functional brain area. Electroencephalogr Clin Neurophysiol Suppl 1996;46:209-13.

8 Rezai AR, Hund M, Kronberg E, et al. The interactive use of magnetoencephalography in stereotactic image-guided neurosurgery. Neurosurgery 1996:39:92-102.

9 Bittar RG, Olivier A, Sadikot AF, et al. Localization of somatosensory function by using positron emission tomography scanning: a comparison with intraoperative cortical stimulation. J Neurosurg 1999;90:478-83.

10 Ganslandt O, Fahlbusch R, Nimsky C, et al. Functional neuronavigation with magnetoencephalography: outcome in 50 patients with lesions around the motor cortex. J Neurosurg 1999;91:73-9.

11 Cedzich C, Taniguchi M, Schafer S, et al. Somatosensory evoked potential phase reversal and direct motor cortex stimulation during surgery in and around the central region. Neurosurgery 1996:38:962-70.

12 Penfield W, Boldrey E. Somatic motor and sensory representation in the cerebral cortex of man as studied by electrical stimulation. Brain 1937;60:389-443

13 Yingling CD, Ojemann S, Dodson B, et al. Identification of motor pathways during tumor surgery facilitated by multichannel electromyographic recording. J Neurosurg 1999;91:922-7.

14 Aiba T, Seki Y. Intraoperative identification of the central sulcus: a practical method. Acta Neurochir Suppl 1988:42:22-6.

15 Babu KS, Chandy M. Reliability of somatosensory evoked potentials in intraoperative localization of the central sulcus in patients with perirolandic mass lesions. Br J Neurosurg 1997;11:411-7.

16 Gregorie EM, Goldring S. Localization of function in the excision of lesions from the sensorimotor region. J Neurosurg 1984;61:1047-54

17 King RB, Schell GR. Cortical localization and monitoring during cerebral operations. J Neurosurg 1987;67:210-9.

18 Nuwer MR, Banoczi WR, Cloughesy TF, et al. Topographic mapping of somatosensory evoked potentials helps identify motor cortex more quickly in the operating room. Brain Topogr 1992;5:53-8.

19 Wood CC, Spencer DD, Allison T, et al. Localization of human sensorimotor cortex during surgery by cortical surface recording of somatosensory evoked potentials. J Neurosurg 1988;68:99-1 11.

20 Woolsey CN, Erickson TC, Gilson WE. Localization in somatic sensory and motor areas of human cerebral cortex as determined by direct recording of evoked potentials and electrical stimulation. J Neurosurg 1979;51:476-506.

21 Paleologos TS, Wadley JP, Kitchen ND, et al. Clinical utility and cost-effectiveness of interactive image-guided craniotomy: clinical comparison between conventional and image-guided meningioma surgery. Neurosurgery 2000;47:40-7.

22 Wirtz CR, Albert FK, Schwaderer M, et al. The benefit of neuronavigation for neurosurgery analyzed by its impact on glioblastoma surgery. Neurol Res 2000;22:354-60.

23 Bittar RG, Olivier A, Sadikot AF, et al. Cortical motor and somatosensory representation: effect of cerebral lesions. J Neurosurg 2000;92:242-8.

24 Sonoo M, Shimpo T, Takeda K, et al. SEPs in two patients with localized lesions of the postcentral gyrus. Electroencephalogr Clin Neurophysiol $1991 ; 80: 536-46$.

25 Suzuki A, Yasui N. Intraoperative localization of the central sulcus by cortical somatosensory evoked potentials in brain tumor. Case report. J Neurosurg 1992;76:867-70.

26 Baumgartner C, Barth DS, Levesque MF, et al. Functional anatomy of human hand sensorimotor cortex from spatiotemporal analysis of electrocorticography. Electroencephalogr Clin Neurophysiol 1991;78:56-65.

27 Lüders H, Lesser RP, Hahn J, et al. Cortical somatosensory evoked potentials in response to hand stimulation. J Neurosurg 1983;58:885-94.

28 Nuwer MR, Aminoff $M$, Desmedt J, et al. IFCN recommended standards for short latency somatosensory evoked potentials. Report of an IFCN committee. International Federation of Clinical Neurophysiology. Electroencephalogr Clin Neurophysiol 1994;91:6-11.
29 Uematsu S, Lesser R, Fisher RS, et al. Motor and sensory cortex in humans: topography studied with chronic subdural stimulation. Neurosurgery 1992;31:59-71

30 Ganslandt O, Steinmeier R, Kober H, et al. Magnetic source imaging combined with image-guided frameless stereotaxy: a new method in surgery around the motor strip. Neurosurgery 1997;41:621-7.

31 Baumgartner C, Barth DS, Levesque MF, et al. Human hand and lip sensorimotor cortex as studied on electrocorticography. Electroencephalogr Clin Neurophysiol 1992;84:115-26.

32 Ebeling U, Schmid UD, Ying $H$, et al. Safe surgery of lesions near the motor cortex using intra-operative mapping techniques: a report on 50 patients. Acta Neurochir 1992;119:23-8.

33 Kombos T, Suess O, Funk T, et al. Intra-operative mapping of the motor cortex during surgery in and around the motor cortex. Acta Neurochir 2000; 142:263-8

34 Allison T, McCarthy G, Wood CC, et al. Potentials evoked in human and monkey cerebral cortex by stimulation of the median nerve. A review of scalp and intracranial recordings. Brain 1991:114:2465-503.

35 Lüders H, Dinner DS, Lesser RP, et al. Evoked potentials in cortical localization. J Clin Neurophysiol 1986;3:75-84

36 Hayashi N, Nishijo H, Ono T, et al. Generators of somatosensory evoked potentials investigated by dipole tracing in the monkey. Neuroscience 1995:68:323-38.

37 Namiki J, Takase M, Ohira T, et al. The neural origin generating early cortical components of SEP: topographical analysis using temporal-second-order-differentiation of cortical SEPs. Brain Topogr 1996;8:229-32.

38 Mine S, Oka N, Yamaura A, et al. Presurgical functional localization of primary somatosensory cortex by dipole tracing method of scalp-skull-brain head model applied to somatosensory evoked potential. Electroencephalogr Clin Neurophysiol 1998;108:226-33.

39 Desmedt JE, Nguyen TH, Bourguet M. Bit-mapped color imaging of human evoked potentials with reference to the N20, P22, P27, and N30 somatosensory responses. Electroencephalogr Clin Neurophysiol 1987:68:1-19

40 Dinner DS, Lüders H, Lesser RP, et al. Cortical generators of somatosensory evoked potentials to median nerve stimulation. Neurology 1987;37:1141-5

41 Nii Y, Uematsu S, Lesser RP, et al. Does the central sulcus divide motor and sensory functions? Cortical mapping of human hand areas as revealed by electrical stimulation through subdural grid electrodes. Neurology 1996;46:360-7

42 Fried I, Nenov VI, Ojemann SG, et al. Functional MR and PET imaging of rolandic and visual cortices for neurosurgical planning. J Neurosurg 1995;83:854-61

43 Puce A. Comparative assessment of sensorimotor function using functional magnetic resonance imaging and electrophysiological methods. J Clin Neurophysiol 1995;12:450-9

44 Uematsu S, Lesser RP, Gordon B. Localization of sensorimotor cortex: the influence of Sherrington and Cushing on the modern concept. Neurosurgery 1992;30:904-12

45 Lehericy S, Duffau H, Cornu P, et al. Correspondence between functional magnetic resonance imaging somatotopy and individual brain anatomy of the central region: comparison with intraoperative stimulation in patients with brain tumors. J Neurosurg 2000;92:589-98.

46 Duffau H, Sichez JP, Lehericy S. Intraoperative unmasking of brain redundant motor sites during resection of a precentral angioma: evidence using direct cortical stimulation. Ann Neurol 2000;47:132-5.

47 Kombos T, Pietila T, Kern BC, et al. Demonstration of cerebral plasticity by intra-operative neurophysiological monitoring: report of an uncommon case. Acta Neurochir 1999;141:885-9.

48 Seitz RJ, Huang Y, Knorr U, et al. Large-scale plasticity of the human motor cortex. Neuroreport 1995;6:742-4.

49 Wunderlich G, Knorr U, Herzog H, et al. Precentral glioma location determines the displacement of cortical hand representation. Neurosurgery 1998:42:18-26.

50 Hund M, Rezai AR, Kronberg E, et al. Magnetoencephalographic mapping: basic of a new functional risk profile in the selection of patients with cortical brain lesions. Neurosurgery 1997;40:936-42.

51 Nimsky C, Ganslandt O, Cerny S, et al. Quantification of, visualization of, and compensation for brain shift using intraoperative magnetic resonance imaging. Neurosurgery 2000:47:1070-9.

52 Inoue T, Shimizu H, Nakasato N, et al. Accuracy and limitation of functional magnetic resonance imaging for identification of the central sulcus: comparison with magnetoencephalography in patients with brain tumors. Neuroimage 1999;10:738-48.

53 Duffau $\mathbf{H}$. Intraoperative direct subcortical stimulation for identification of the internal capsule, combined with an image-guided stereotactic system during surgery for basal ganglia lesions. Surg Neurol 2000;53:250-4

54 Pechstein U, Cedzich C, Nadstawek J, et al. Transcranial high-frequency repetitive electrical stimulation for recording myogenic motor evoked potentials with the patient under general anesthesia. Neurosurgery 1996;39:335-43

55 Kaus MR, Nabavi A, Mamisch CT, et al. Simulation of corticospinal tract displacement in patients with brain tumors. In: Delp SL, DiGioia AM, Jaramaz B, eds. Medical image computing and computer-assisted intervention: proceedings of the third international conference - MICCAI 2000. Berlin Heidelberg New York: Springer, 2000:9-18. 